\title{
Microscopic dynamics of supercooled liquids from first principles
}

\author{
Liesbeth M. C. Janssen 1, * and David R. Reichman, 1, 丹 \\ ${ }^{I}$ Department of Chemistry, Columbia University, 3000 Broadway, New York, New York 10027, USA
}

(Dated: August 20, 2021)

\begin{abstract}
The transition from a liquid to a glass remains one of the most poorly understood phenomena in condensed matter physics, and still no fully microscopic theory exists that can describe the dynamics of supercooled liquids in a quantitative manner over all relevant time scales. Here we present a theoretical framework that yields near-quantitative accuracy for the time-dependent correlation functions of a glass-forming system over a broad density range. Our approach requires only simple static structural information as input and is based entirely on first principles. Owing to its ab initio nature, the framework offers a unique platform to study the relation between structure and dynamics in glass-forming matter, and paves the way towards a systematically correctable and ultimately fully quantitative theory of microscopic glassy dynamics.
\end{abstract}

Understanding the dynamics of supercooled liquids represents one of the major challenges in condensed matter science [1]. Perhaps the most striking feature of vitrification is the observed dramatic increase in viscosity (or relaxation time) upon only a relatively mild change in thermodynamic control parameters, e.g. temperature or density [2]. This highly nonlinear response of the system is further accompanied by only subtle changes in the microscopic structure, thus raising the question as to what physical mechanism underlies the glass transition [3, 4].

Among the various theories of the glass transition proposed in the last few decades [5], mode-coupling theory (MCT) has acquired a prominent place in this field of research [6-8]. MCT is the only strictly first-principles theory of glassy dynamics, and it can accurately predict many features of the time-dependent dynamics of supercooled liquids from simple static information alone, including multi-step relaxation patterns [6], stretched exponentials [6], and growing dynamical length scales [9]. MCT forms a mean-field framework for broader theories such as Random First-Order Theory (RFOT) [10], but it remains a major open question how the relaxation scenarios posited in RFOT can be captured from first principles and fully microscopically along the same lines as MCT. Thus far, efforts to systematically correct the MCT equations, e.g. via higher order field-theoretic loop expansions [11-14], have not been explicitly carried out in a practical setting. Hence, unlike the situation that arises in fields such as electronic structure theory, where the correlation energy can be systematically captured by building upon Hartree-Fock theory [15], there exists no means of providing a more accurate description of glassy dynamics by microscopically correcting the lowest-level mean-field approach. Indeed, a commonly espoused viewpoint is that there exists a "no-theory region" between MCT and the true glassy regime [16]. In this work we show that such a theory can in fact be formulated and suc-

\footnotetext{
${ }^{*}$ Current address: Institute for Theoretical Physics II, Heinrich-Heine University Düsseldorf, Universitätsstraße 1, D-40225, Germany; Electronic mail: ljanssen@thphy.uni-duesseldorf.de

${ }^{\dagger}$ Electronic mail: drr2103@columbia.edu
}

cessfully carried out, and we present comparisons with dynamics in a realistic model of a glassy hard-sphere suspension to quantify the systematic improvement achieved with our approach.

The key quantity in our discussion is the two-point density correlation function $F(k, t)=N^{-1}\left\langle\rho_{-\mathbf{k}}(0) \rho_{\mathbf{k}}(t)\right\rangle$, which probes correlated particle motion over time $t$ and at inverse length scale $k$. Here $\rho_{\mathbf{k}}(t)$ represents a density mode at time $t$ and wavevector $\mathbf{k}, k=|\mathbf{k}|, N$ is the total number of particles, and the brackets denote a canonical ensemble average. The exact time evolution of $F(k, t)$ is governed by an integrodifferential equation with memory function $M(k, t)$, the latter of which accounts for time-dependent damping effects [6]. More precisely, $M(k, t)$ is related to the autocorrelation function of a fluctuating force, which captures the effect of all degrees of freedom orthogonal to the density field [3]. It can be shown that this fluctuating force is dominated by pairs of density modes $\rho_{\mathbf{q}} \rho_{\mathbf{k}-\mathbf{q}}$, allowing the memory function to be expressed in terms of four-point density correlation functions $\left\langle\rho_{-\mathbf{q}}(0) \rho_{\mathbf{q}-\mathbf{k}}(0) \rho_{\mathbf{q}^{\prime}}(t) \rho_{\mathbf{k}-\mathbf{q}^{\prime}}(t)\right\rangle$ [6] [34]. Within the traditional MCT framework, the four-point correlation functions are subsequently approximated as products of two two-point correlation functions, thereby rendering a closed, non-linear equation of motion for $F(k, t)[6,8]$. The microscopic structure of the system enters through the static structure factor $S(k) \equiv F(k, 0)$ [17], after Gaussian and convolution approximations are made for the statics.

In the present work, we seek to avoid the uncontrolled 'Gaussian' factorization of the dynamic four-point correlations, instead retaining these correlations in the expression for $F(k, t)$ and developing a new exact equation of motion for the memory term. As shown explicitly in the Supplementary Information (SI) [35], the dynamics of these fourpoint correlation functions is governed by a new memory kernel that (after projection onto triplet-density modes) contains six-point correlations, i.e. correlation functions of the form $\left\langle\rho_{\mathbf{k}_{1}}(0) \rho_{\mathbf{k}_{2}}(0) \rho_{\mathbf{k}_{3}}(0) \rho_{\mathbf{k}_{1}^{\prime}}(t) \rho_{\mathbf{k}_{2}^{\prime}}(t) \rho_{\mathbf{k}_{3}^{\prime}}(t)\right\rangle$. These are controlled by eight-point correlations, and so on, thus allowing one to delay the uncontrolled factorization approximation to a later stage. Our approach builds upon the important work of Szamel, who first introduced such a hierarchical scheme of microscopic kinetic equations [18]. Several recent stud- 
ies have demonstrated that this approach indeed holds great potential as a microscopic theory of glassy dynamics. In particular, it was shown that higher-order correlations can systematically improve the predicted MCT critical point [18, 19]. Moreover, two schematic ( $k$-independent) proof-of-principle studies predicted a wealth of novel relaxation patterns for continuous, discontinuous, and avoided glass transitions [20, 21]. For example, the theory may describe both Arrhenius and super-Arrhenius growth of the relaxation time [21], thus providing potentially the first rigorous first-principles rationale for the concept of fragility [2, 22].

Here we report on the first fully microscopic, high-order calculations to describe the explicit time- and $k$-dependent dynamics of a realistic glassy system. We focus on a system composed of quasi-hard spheres, and compare our theoretical predictions with the exact dynamics obtained from computer simulations [23]. By using only the simplest measure of static correlations, i.e. $S(k)$, as sole input to our theory, we already achieve high quantitative accuracy for $F(k, t)$, for all wavevectors, in the regime from low to moderate supercooling. In view of its first-principles nature, the theory can shed important new light on the fundamental connection between structure and dynamics in glass-forming matter, and paves the way towards an ultimately fully quantitative description of glassy dynamics.

The first main result of this work is the formulation of a new set of microscopic equations of motion for the dynamic density correlation functions of a glass-forming system. The full derivation is described in the SI. We consider the dynamics of the normalized $2 n$-point density correlation functions $\Phi_{n}\left(k_{1}, \ldots, k_{n}, t\right)$, which probe particle correlations over $n$ distinct $k$-values,

$\Phi_{n}\left(k_{1}, \ldots, k_{n}, t\right)=\frac{\left\langle\rho_{-\mathbf{k}_{1}}(0) \ldots \rho_{-\mathbf{k}_{\mathbf{n}}}(0) \rho_{\mathbf{k}_{1}}(t) \ldots \rho_{\mathbf{k}_{\mathbf{n}}}(t)\right\rangle}{\left\langle\rho_{-\mathbf{k}_{1}}(0) \ldots \rho_{-\mathbf{k}_{\mathbf{n}}}(0) \rho_{\mathbf{k}_{1}}(0) \ldots \rho_{\mathbf{k}_{\mathbf{n}}}(0)\right\rangle}$.

Within our framework, these correlators obey the general equations of motion

$$
\begin{gathered}
\ddot{\Phi}_{n}\left(k_{1}, \ldots, k_{n}, t\right)+\nu \dot{\Phi}_{n}\left(k_{1}, \ldots, k_{n}, t\right) \\
+\Omega_{n}^{2}\left(k_{1}, \ldots, k_{n}\right) \Phi_{n}\left(k_{1}, \ldots, k_{n}, t\right) \\
+\int_{0}^{t} M_{n}\left(k_{1}, \ldots, k_{n}, \tau\right) \dot{\Phi}_{n}\left(k_{1}, \ldots, k_{n}, t-\tau\right) d \tau=0,
\end{gathered}
$$

where the dots denote time derivatives, $\nu$ represents a coefficient that accounts for the short-time dynamics, and the bare frequencies are given by

$$
\Omega_{n}^{2}\left(k_{1}, \ldots, k_{n}\right)=\frac{k_{\mathrm{B}} T}{m}\left[\frac{k_{1}^{2}}{S\left(k_{1}\right)}+\ldots+\frac{k_{n}^{2}}{S\left(k_{n}\right)}\right]
$$

Here $k_{\mathrm{B}}$ is the Boltzmann constant, $T$ is the temperature, and $m$ is the particle mass. For the memory functions we have

$$
\begin{aligned}
& M_{n}\left(k_{1}, \ldots, k_{n}, t\right)=\frac{\rho k_{\mathrm{B}} T}{16 m \pi^{3}} \sum_{i=1}^{n} \frac{\Omega_{1}^{2}\left(k_{i}\right)}{\Omega_{n}^{2}\left(k_{1}, \ldots, k_{n}\right)} \\
& \times \int d \mathbf{q}\left|\tilde{V}_{\mathbf{q}, \mathbf{k}_{\mathbf{i}}-\mathbf{q}}\right|^{2} S(q) S\left(\left|\mathbf{k}_{\mathbf{i}}-\mathbf{q}\right|\right) \\
& \times \Phi_{n+1}\left(q,\left|\mathbf{k}_{\mathbf{1}}-\mathbf{q} \delta_{i, 1}\right|, \ldots,\left|\mathbf{k}_{\mathbf{n}}-\mathbf{q} \delta_{i, n}\right|, t\right),
\end{aligned}
$$

where $\rho$ is the total density, $\delta_{i, j}$ is the Kronecker delta function, and $\tilde{V}_{\mathbf{q}, \mathbf{k}_{\mathbf{i}}-\mathbf{q}}$ are static vertices that represent wavevectordependent coupling strengths for the higher-order correlations. These vertices depend on the microscopic structure of the system contained in $S(k)$, which serves as input to the theory [8]. Note that the memory function at level $n$ contains $n$ different terms, each of which contains a $2(n+1)$ point correlator that measures correlations over a distinct set of wavenumbers $\left\{q, k_{1}, \ldots,\left|\mathbf{k}_{\mathbf{i}}-\mathbf{q}\right|, k_{i+1}, \ldots, k_{n}\right\}$. In arriving at the above equations, we have retained all diagonal dynamic $2 n$-point correlation functions, and employed Gaussian and convolution approximations for the static correlators (see SI). These remaining (uncontrolled) assumptions are essentially the same as at the standard-MCT level; the crucial difference between our work and standard MCT is that we do not factorize the diagonal dynamic multi-point correlators. We expect the inclusion of off-diagonal dynamic correlators to lead to a matrix form of the hierarchy that is likely to be far more demanding computationally, but identical in overall structure compared to the present set of equations. We may close this hierarchy of coupled equations at an arbitrary level $n=N$ by applying a 'mean-field' (MF) factorization of the form $\Phi_{N}(t) \sim \Phi_{N-1}(t) \Phi_{1}(t)$ [the simplest example of which is the standard-MCT closure $\left.\Phi_{2}(t) \sim \Phi_{1}(t) \Phi_{1}(t)\right]$ or by simply setting $\Phi_{N}(t)=0$. The latter implies that $\Phi_{N-1}(t) \sim \exp \left(-\Omega_{N-1}^{2} t\right)$, hence we refer to this truncation as an exponential closure. Finally, we emphasize that our theory is free of any fitting or rescaling parameters, thus providing dynamic predictions from purely static information alone.

In Fig. 11 we show our numerical results for the density correlator $\Phi_{1}(k, t)=F(k, t) / S(k)$ for the quasi-hard sphere system of Ref. [23]. While this system is slightly polydisperse to prevent crystallization, we have used the averaged (effectively monodisperse) structural properties as input to our calculations, as was also employed by the authors of Ref. [23]. As a stringent test of our theory, we compare the theoretically predicted dynamics with the numerically simulated $\Phi_{1}(k, t)$ for different wavevectors and different volume fractions $\phi=\rho \pi d^{3} / 6$, where $d$ is the average particle diameter. Note that, while our theory also readily gives access to the higher-order correlators, the simulated data are limited to $\Phi_{1}(k, t)$. The wavevectors we consider correspond to the main $(k d=7.4)$ and second $(k d \approx 13.0)$ peak of the static structure factor. We have solved the time-dependent coupled equations numerically employing both MF and exponential 

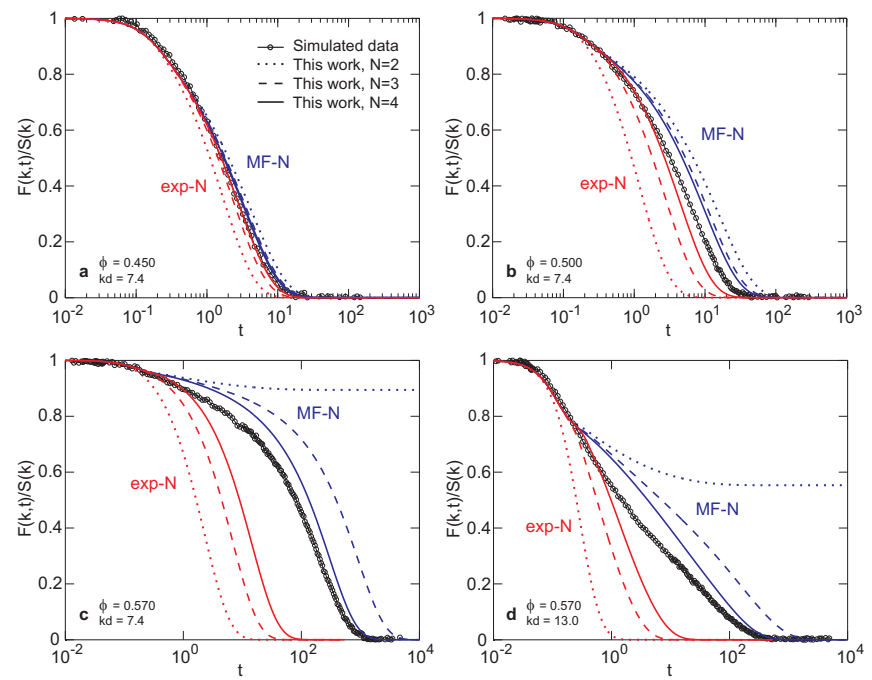

FIG. 1: Two-point density correlation functions $\Phi_{1}(k, t)$ for quasihard spheres at different volume fractions and wavevectors: (a), $\phi=$ $0.450, k d=7.4,(\mathbf{b}), \phi=0.500, k d=7.4,(\mathbf{c}), \phi=0.570$, $k d=7.4,(\mathbf{d}), \phi=0.570, k d=13.0$. The theoretical results have been obtained using either exponential (red lines) or MF (blue lines) closures, with closure levels $N=2,3$, and 4 . The $N=2 \mathrm{MF}$ closure is equivalent to standard MCT. All legends are as in panel (a). The simulated data (black circles) are taken from Ref. [23].

closures for truncation levels $N=2,3,4$. Computational details can be found in the SI.

Before discussing Fig. 11in detail, we first point out an important general observation with regard to the convergence of our results. For all volume fractions and wavevectors considered, we find that the MF closures provide an upper bound to the dynamics, i.e. the $N=2$ (standard-MCT) closure predicts the slowest relaxation, while the exponential closures give a lower bound to the dynamics. This is expected because any mean-field theory will generally underestimate the effects of ergodicity-restoring fluctuations, consequently overestimating the dynamic slowing down. Conversely, an exponential closure rigorously ignores higher-order memory effects that may drive the system into the glassy state [since $\Phi_{N}(t) \sim M_{N+1}(t)=0$ ], resulting in relaxation patterns that are too fast. With increasing closure level $N$, however, the two types of closures systematically converge towards each other over an increasingly large time domain. This uniform convergence pattern can be rigorously demonstrated within the confines of a schematic model [20], and provides a unique and clearly achievable notion of convergence even though no small parameter exists in our approach.

Let us now focus on the time-dependent relaxation behavior of the quasi-hard sphere system for specific values of $\phi$. For the lowest volume fraction considered, $\phi=0.450$, the system is still strongly ergodic and $\Phi_{1}(k, t)$ exhibits relatively simple relaxation [Fig. [1a)]. Here our $N=4$ data are almost perfectly converged upon the simulated $\Phi_{1}(k, t)$ curve over the complete time domain. Note that the standard-MCT result is also reasonably accurate, but slightly overestimates the molec- ular relaxation time. These findings hold for all wavevectors considered. Thus, in the "normal liquid" regime, the predictions of our higher-order theory are virtually exact with respect to computer simulations.

For a higher volume fraction, $\phi=0.500$, the relaxation time is seen to increase and the system enters the supercooled regime [Fig. 1 b)]. Again we find nearly perfect quantitative agreement between our $N=4$ results and the simulated density correlator at $k d=7.4$, indicating that our higherorder microscopic approach accurately captures the onset of glassy dynamics at the correct absolute value of $\phi$. This is to be contrasted with standard MCT $(N=2)$, which clearly overestimates the glassiness of the system and can only reproduce the simulated results for a rescaled volume fraction $\phi^{\mathrm{MCT}}<0.500$ [23]. Since the higher-order theory presented here does not require such rescaling of the control parameter, the inclusion of higher-order dynamic correlations thus appears to capture all relevant relaxation mechanisms in the weakly supercooled regime.

We now turn our attention to the highest volume fraction, $\phi=0.570$, where the system is significantly supercooled. Standard MCT predicts a diverging relaxation time above $\phi_{c}^{\mathrm{MCT}}=0.566$ [23], as seen in our $N=2$ results in Figs. 1 (c)-(d). The simulated $\Phi_{1}(k, t)$ curves, however, show complete relaxation to zero, indicating that the system is still ergodic at this density. In agreement with these simulations, our $N=3$ and $N=4$ results also predict full relaxation, thus restoring ergodicity and rounding off the spurious MCT transition. We find good agreement with the simulated data for both wavevectors, but our higher-order theoretical results appear to converge toward a relaxation time that is slightly too fast compared to the simulations. The predicted curves for $k d=13.0$ also develop a small shoulder at intermediate times that is absent in the simulated data. This slightly poorer agreement for $k d>7.4$ was also noted by Weysser et al. [23] for the standard-MCT case, even after rescaling of the data. The origin of the discrepancy may be related to the fact that the use of a density-mode basis neglects some short-time correlations, which are expected to be more pronounced away from the first peak of $S(k)$. We attribute further discrepancies to the remaining approximations in our theory, i.e. the neglect of off-diagonal dynamic correlation functions $\left\langle\rho_{\mathbf{q}}(0) \ldots \rho_{\mathbf{q}^{\prime}}(t)\right\rangle$ with $\mathbf{q} \neq \mathbf{q}^{\prime}$, and the factorization of the static multi-point correlations. Indeed, one intriguing possibility is that the weaker agreement with simulation at the highest densities indicates that multi-point static correlations, such as the much studied point-to-set correlations [3], begin to extend a substantial influence on the relaxation. In this regard, our approach also has utility in delineating the role of static structural properties beyond $S(k)$ on the dynamics. Finally, Weysser et al. have found that the standard-MCT predictions for this system can be improved, in particular for small $k$-values, by accounting for polydispersity effects [23]. Our theoretical results may thus also be further improved by careful treatment of the system's polydispersity.

Despite the quantitative differences found in Fig.1(c)-(d), 

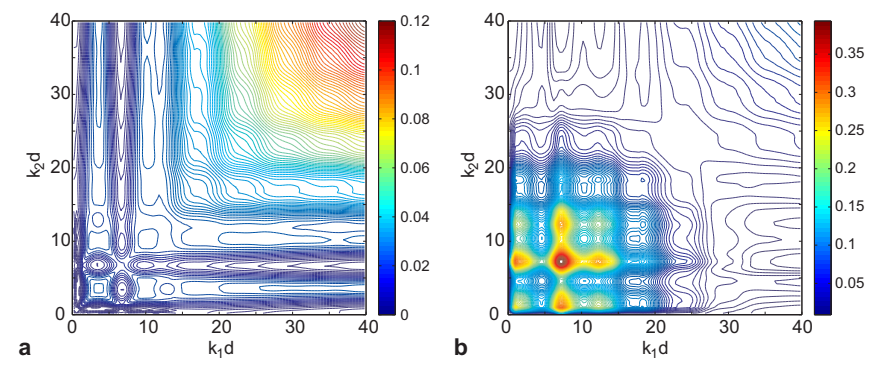

FIG. 2: Root-mean-square deviations between the four-point density correlator $\Phi_{2}\left(k_{1}, k_{2}, t\right)$, as obtained from our high-order calculations under an $N=4 \mathrm{MF}$ closure, and the standard-MCT approximation $\Phi_{1}^{\mathrm{MCT}}\left(k_{1}, t\right) \Phi_{1}^{\mathrm{MCT}}\left(k_{2}, t\right)$, as a function of wavevectors $k_{1}$ and $k_{2}$ at (a) $\phi=0.450$ and (b) $\phi=0.570$.

it is evident that our higher-order framework in its current form captures a significant portion of the activated dynamics beyond the standard-MCT scenario, at least up to moderate volume fractions. We emphasize that our theoretical predictions are free from any fitting or rescaling procedures, and that regular MCT would not be able to achieve a similar degree of accuracy, even after introducing a rescaling parameter. The good agreement we find between our work and simulations is highly non-trivial, considering that the only input to our theory is $S(k)$, the simplest measure of the microscopic structure of the system. Our results thus support the view that activated dynamics-at least in the weak to moderate regime of supercooling-can be described via a strictly dynamic framework, in the form of deeper memory effects that occur over increasingly many wavelengths.

As a final part of our analysis, let us elaborate on how our theory improves upon the standard-MCT framework in terms of the memory function. Recall that standard MCT approximates the lowest-order memory contribution $\Phi_{2}(q,|\mathbf{k}-\mathbf{q}|, t)$ as $\Phi_{2}^{\mathrm{MCT}}(q,|\mathbf{k}-\mathbf{q}|, t) \sim \Phi_{1}^{\mathrm{MCT}}(q, t) \Phi_{1}^{\mathrm{MCT}}(|\mathbf{k}-\mathbf{q}|, t)$ [8]. Conversely, our framework retains the four-point correlations explicitly and treats their dynamics rigorously through a hierarchy of equations. Understanding how the $\Phi_{2}(q,|\mathbf{k}-\mathbf{q}|, t)$ that emerges from our approach differs from $\Phi_{2}^{\mathrm{MCT}}(q,|\mathbf{k}-\mathbf{q}|, t)$ thus provides insight into the improvements afforded over standard MCT. In Fig. 2] we compare our best estimate $(N=4)$ for the dynamics of the four-point correlation function $\Phi_{2}\left(k_{1}, k_{2}, t\right)$ with the standard-MCT approximation $\Phi_{1}^{\mathrm{MCT}}\left(k_{1}, t\right) \Phi_{1}^{\mathrm{MCT}}\left(k_{2}, t\right)$. We plot the root-mean-square deviation (RMSD) $\mid \Phi_{2}\left(k_{1}, k_{2}, t\right)-$ $\Phi_{1}^{\mathrm{MCT}}\left(k_{1}, t\right) \Phi_{1}^{\mathrm{MCT}}\left(k_{2}, t\right) \mid / \sqrt{N_{t}}$, where $N_{t}$ is the total number of time points, for volume fractions $\phi=0.450$ and $\phi=0.570$. It may be seen that for both volume fractions the RMSD is modulated by the structure of $S(k)$. In the lowdensity regime $[\phi=0.450$, Fig. 2 $($ a) $]$, the largest deviations are found at large wavevectors. These differences arise from the interplay between the bare frequency term $\Omega_{2}\left(k_{1}, k_{2}\right)$ and the inertial term $\ddot{\Phi}_{2}\left(k_{1}, k_{2}, t\right)$, which make the four-point correlation functions $\Phi_{2}\left(k_{1}, k_{2}, t\right)$ oscillate and become negative at short times. Such negative contributions are mostly absent in standard MCT, since the diagonal MCT terms $\Phi_{2}(k, k, t) \sim$ $\left|\Phi_{1}(k, t)\right|^{2}$ are always positive. Hence, standard MCT tends to overestimate the memory term $M_{1}(k, t)$ and consequently overestimates the relaxation time. These oscillatory patterns only arise in the underdamped limit, however, and neglecting the inertial term (i.e. overdamped dynamics) dramatically reduces the RMSD in the large-wavevector regime.

In the deeply supercooled region $[\phi=0.570$, Fig. 2(b)], the overall RMSD increases, and the largest deviation is now observed at smaller wavevectors, most notably near the main peak of $S(k)$ at $k_{1} d \approx k_{2} d \approx 7.4$. The RMSD also exhibits local maxima at wavevectors of $k d \approx 13.0$, corresponding to the second peak of the structure factor, and $k d \approx 1.0$. Thus, the ergodicity-restoring processes that round off the standardMCT transition are mainly contained in the lower- $k$ behavior of the memory function. It is well established that the standard-MCT framework is generally the least accurate in the hydrodynamic $k \rightarrow 0$ limit, resulting in e.g. an underestimation of Stokes-Einstein violation in the deeply supercooled regime [24]. Since the higher-order corrections at large volume fractions occur mostly at small $k$-values, one may expect that our theory might improve the predicted breakdown of the Stokes-Einstein relation and other related quantities. This possibility will be tested in future work.

In conclusion, we have presented a novel first-principles theory to describe the microscopic dynamics of glassy materials with near-quantitative accuracy in the low to moderately supercooled regime. The framework requires only static structure as input and has no free parameters. Our results demonstrate that a quantitative account of dynamical correlations may be generated from only two-body statics-even in a regime where many-body statics and dynamical heterogeneity are markedly growing-, thus shedding light on the connection between structure and dynamics in supercooled liquids. Our framework may also eventually be used to provide predictive insights into regimes of glassy behavior inaccessible to molecular-dynamics simulations. Not only does our method give straightforward access to e.g. long times and higher-order correlation functions, it also suggests that by incorporating increasingly many (static and dynamic) correlations, one may achieve systematically improvable and ultimately fully quantitative accuracy in the deeply supercooled regime.

As a final note, it should be remarked that the hierarchy presented here resembles the Martin-Schwinger hierarchy [25, 26] in field theory, where low-order Green's functions are hierarchically connected to higher-order ones through an infinite set of coupled equations. In the context of the present work, we point out that there has been substantial recent progress in the formulation of field-theoretic approaches for the glass transition that allow for a systematic inclusion of higher-order terms that are omitted from standard-MCT-like theories $[27-29]$. It is likely that such approaches can be formulated in a Martin-Schwinger-type form, which would enable the direct comparison of the hierarchy presented here with recent field-theoretic formulations. Such a comparison might also make clearer the effect of several approximations 
that have been made here. Future work should be devoted to such a program.

We are greatly indebted to the authors of Ref. [23], in particular Antonio M. Puertas and Matthias Fuchs, for generously making available the results of their simulations to us. LMCJ gratefully acknowledges support from the Netherlands Organization for Scientific Research (NWO) through a Rubicon fellowship. DRR ackowledges grant NSF-CHE 1464802 for support.

[1] P. W. Anderson, Science 267, 1615 (1995).

[2] P. G. Debenedetti and F. H. Stillinger, Nature 410, 259 (2001).

[3] L. Berthier and G. Biroli, Rev. Mod. Phys. 83, 587 (2011).

[4] C. P. Royall and S. R. Williams, Phys. Rep. 560, 1 (2015).

[5] G. Tarjus, in L. Berthier, G. Biroli, J.-P. Bouchaud, L. Cipelletti, and W. van Saarloos, eds., Dynamical Heterogeneities in Glasses, Colloids, and Granular Media (Oxford University Press, Oxford, 2011), p. 39.

[6] W. Götze, Complex dynamics of glass-forming liquids: A mode-coupling theory (Oxford University Press, Oxford, 2009).

[7] S. P. Das, Rev. Mod. Phys. 76, 785 (2004).

[8] D. R. Reichman and P. Charbonneau, J. Stat. Mech. p. P05013 (2005).

[9] G. Biroli, J. Bouchard, K. Miyazaki, and D. R. Reichman, Phys. Rev. Lett. 97, 195701 (2006).

[10] T. R. Kirkpatrick, D. Thirumalai, and P. G. Wolynes, Phys. Rev. A 40, 1045 (1989).

[11] S. P. Das and G. F. Mazenko, Phys. Rev. A 34, 2265 (1986).

[12] W. Götze and L. Sjögren, Z. Phys. B 65, 415 (1987).

[13] A. Andreanov, G. Biroli, and A. Lefèvre, J. Stat. Mech. p. P07008 (2006).

[14] K. Miyazaki and D. R. Reichman, J. Phys. A 38, L343 (2005).

[15] T. Helgaker, P. Jorgensen, and J. Olsen, Molecular
Electronic-Structure Theory (Wiley, Chichester, 2000).

[16] J. S. Langer, Rep. Prog. Phys. 77, 042501 (2014).

[17] J.-P. Hansen and I. R. McDonald, Theory of Simple Liquids (Elsevier Academic Press, 2006), 3rd ed.

[18] G. Szamel, Phys. Rev. Lett. 90, 228301 (2003).

[19] J. Wu and J. Cao, Phys. Rev. Lett. 95, 078301 (2005).

[20] P. Mayer, K. Miyazaki, and D. R. Reichman, Phys. Rev. Lett. 97, 095702 (2006).

[21] L. M. C. Janssen, P. Mayer, and D. R. Reichman, Phys. Rev. E 90, 052306 (2014).

[22] C. A. Angell, Science 267, 1924 (1995).

[23] F. Weysser, A. M. Puertas, M. Fuchs, and T. Voigtmann, Phys. Rev. E 82, 011504 (2010).

[24] L. Berthier, Phys. Rev. E 69, 020201 (2004).

[25] P. C. Martin and J. Schwinger, Phys. Rev. 115, 1342 (1959).

[26] J. W. Negele and H. Orland, Quantum Many-Particle Systems (Westview Press, 1998).

[27] S. P. Das and G. F. Mazenko, J. Stat. Phys. 149, 643 (2012).

[28] S. P. Das and G. F. Mazenko, J. Stat. Phys. 152, 159 (2013).

[29] D. D. McCowan, Phys. Rev. E 92, 022107 (2015).

[30] J. Schofield, R. Lim, and I. Oppenheim, Physica A 181, 89 (1992).

[31] W. Kob, in J. Fourkas, D. Kivelson, U. Mohanty, and K. Nelson, eds., Experimental and Theoretical Approaches to Supercooled Liquids: Advances and Novel Applications (ACS Books, Washington, 1997), p. 28.

[32] T. Franosch, M. Fuchs, W. Götze, M. R. Mayr, and A. P. Singh, Phys. Rev. E 55, 7153 (1997).

[33] M. Fuchs, W. Götze, I. Hofacker, and A. Latz, J. Phys.: Condens. Matter 3, 5047 (1991).

[34] The time dependence of this four-point correlation function occurs in a subspace orthogonal to the single density mode. However, because the pair-densities can be made orthogonal to the linear density [30], it is sensible in further approximations to treat the evolution with unprojected dynamics.

[35] See Supplemental Material [url], which includes Refs. [31-33]. 